\title{
Effects of cocktail of four local Malaysian medicinal plants (Phyllanthus spp.) against dengue virus 2
}

Sau Har Lee ${ }^{1}$, Yin Quan Tang ${ }^{1}$, Anusyah Rathkrishnan ${ }^{1}$, Seok Mui Wang ${ }^{2}$, Kien Chai Ong ${ }^{3}$, Rishya Manikam Bobby Joe Payne ${ }^{5}$, Indu Bala Jaganath ${ }^{6}$ and Shamala Devi Sekaran ${ }^{1 *}$

\begin{abstract}
Background: The absence of commercialized vaccines and antiviral agents against dengue has made the disease a major health concern around the world. With the current dengue virus transmission rate and incidences, the development of antiviral drugs is of vital need. The aim of this project was to evaluate the possibility of developing a local medicinal plant, Phyllanthus as an anti-dengue agent.
\end{abstract}

Methods: Cocktail (aqueous and methanolic) extracts were prepared from four species of Phyllanthus (P.amarus, P.niruri, P.urinaria, and P.watsonii) and their polyphenolic compounds were identified via HPLC and LC-MS/MS analysis. MTS assay was then carried out to determine the maximal non-toxic dose (MNTD) of the extracts, followed by screening of the in vitro antiviral activity of aqueous cocktail extracts against DENV2 by means of time-of -addition (pre-, simultaneous and post-) using RT-qPCR. The differentially expressed proteins in the treated and infected cells were analysed with two dimensional gel electrophoresis experiments.

Results: Several active compounds including gallic acid, geraniin, syringin, and corilagen have been identified. The MNTD of both aqueous and methanolic extracts on Vero cells were $250.0 \mu \mathrm{g} / \mathrm{ml}$ and $15.63 \mu \mathrm{g} / \mathrm{ml}$ respectively. Phyllanthus showed strongest inhibitory activity against DENV2 with more than $90 \%$ of virus reduction in simultaneous treatment. Two-dimensional analysis revealed significantly altered levels of thirteen proteins, which were successfully identified by tandem MS (MS/MS). These altered proteins were involved in several biological processes, including viral entry, viral transcription and translation regulations, cytoskeletal assembly, and cellular metabolisms.

Conclusions: Phyllanthus could be potentially developed as an anti-DENV agent.

Keywords: Phyllanthus, Dengue, Antiviral

\section{Background}

Dengue virus (DENV), of the family Flaviviridae, is the causative agent for the high morbidity rated diseasedengue. Being a tropical/sub-tropical disease, dengue is currently endemic in more than 100 countries around the world. It is estimated that 36 million dengue fever cases and another 2.1 million cases of severe dengue occur yearly [1]. These numbers are predicted to increase over the years, mainly due to global warming and

\footnotetext{
* Correspondence: shamalamy@yahoo.com

'Department of Medical Microbiology, Faculty of Medicine, University Malaya, Kuala Lumpur, Malaysia

Full list of author information is available at the end of the article
}

the increased frequencies of migration, local and international travelling [2] as this arthropod-borne virus is mainly transmitted by mosquitoes, Aedes aegypti and Aedes albopictus.

DENV is a single-stranded positive sense RNA virus with a genome of approximately $11 \mathrm{~kb}$ [3] which encodes for 3 structural proteins (capsid (C), envelope (E) and membrane $(\mathrm{M}))$ and 7 non-structural (NS) proteins (NS1, NS2A, NS2B, NS3, NS4A, NS4B and NS5). Despite extensive research on dengue, many ambiguities lie in the functions on the various DENV proteins. Nevertheless, the NS3 protein has been shown to be multifunctional with protease activity at its $\mathrm{N}$-terminal

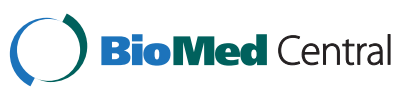


together with NS2B and RNA helicase, a nucleoside triphosphatase, and RNA 5'-triphosphatase at its Cterminal [3,4], while, the N-terminal of NS5 represents a methyltransferase and its C-terminal a RNA-dependent RNA polymerase. NS1, on the other hand, has been suggested to play a role in endothelium dysfunction in dengue [5].

Traditionally classified as dengue fever (DF), dengue haemorrhagic fever (DHF) and dengue shock syndrome (DSS), at present, the disease classification has been revised [6] into dengue with/without warning signs and severe dengue. The usual symptoms of dengue include fever, nausea, rashes, whereas the warning signs for the disease includes abdominal pain, persistent vomiting, mucosal bleeding, hepatomegaly and increased levels of hematocrit concurrent with the decreased levels of platelets. Patients who suffer from severe plasma leakage, haemorrhage and organ impairment have higher mortality rates, if not managed well clinically. A primary infection of dengue confers life-long protection towards that particular serotype, not towards the other 3 DENV serotypes [2]. This particularly complicates secondary infections, where antibody enhancement [7] and crossreactive memory $\mathrm{T}$ cell activation [8] are postulated to cause more severe manifestations of dengue. To date, there are neither effective anti-dengue agents nor commercially licensed vaccines for the treatment of dengue $[9,10]$. The current methods of controlling dengue are mosquito eradication programs [11] and fluid management therapy for infected individuals [6]. Therefore, the need for new antiviral agents is imperative for early treatment to prevent manifestation of severe dengue, to curb outbreaks and in the future to complement possible vaccination programs.

Plants have always been an exclusive part in traditional healing, and in the past decades as treatment sources for various diseases, due to their complex bioactive ingredients and rich source of pharmaceuticals [12]. Plants have been revealed to offer better sources of antiviral agents compared to synthetic analogues [13], which has since prompted identification of more than 3800 plants with efficient abilities to suppress the growth of numerous viruses [14]. These include Olea eurolaea leaf extract which demonstrated strong antiviral activity towards human immunodeficiency virus (HIV) type 1 [15] and influenza virus [16], Rozites caperata which acts against herpes simplex virus (HSV) types 1 and 2 [12], Sargassum patens acting against HSV type 1 [17], as well as Phyllanthus nanus, Salvia miltiorrhiza, Radix astragala, and Rheum palmatum which have anti-hepatitis B virus effect [18]. The most intriguing fact of using plant sources as antiviral agents, lies in the fact that these plants are usually found in abundance in developing and third world countries, where infectious diseases occur are more rampant. One such plant genus is the Phyllanthus (Euphorbiaceae), which has around 750 species [19] and is distributed throughout the tropical and subtropical countries (which are also where dengue occurs). Phyllanthus is well known for its pharmacological properties in folk medicine [20] as well as in modern treatments of kidney and liver diseases, urinary bladder and intestinal infections; diabetes, hepatitis, dysentery, jaundice, gonorrhea and skin ulcers $[20,21]$. The plant has also been proposed to show anti-cancer activities [22,23].

With tremendous potential as pharmacological sources, we seek to evaluate the antiviral potential of Phyllanthus cocktail extracts which is a mixture of four Phyllanthus species, namely $P$. urinaria, $P$. niruri, $P$. amarus, and $P$. watsonii in vitro against dengue virus type 2 (DENV2) as well as to determine its possible pharmacological activity.

\section{Methods}

\section{Cocktail extract preparation}

The cocktail (aqueous and methanolic) extracts consisting of four different pooled Phyllanthus species (P.amarus, P.niruri, P.urinaria, and P.watsonii) were kindly prepared and provided by the Biotechnology Centre, Malaysian Agricultural \& Research Development Institute (MARDI). All four species of Phyllanthus were grown in greenhouse from year January 2008 till December 2010 under controlled irrigation and fertilization at MARDI and the samples were harvested every two months since March 2008. The plant species were identified based on taxonomical identification by Dr Salma Idris, a taxonomist from Strategic Research Centre, MARDI and the herbarium specimen is kept at MARDI, Serdang. Each of the species contains different chemical profiles in terms of the type of phytochemicals and their amount. Hence, a cocktail Phyllanthus extract was prepared from P.watsonii, $P$. amarus, $P$. niruri, and $P$. urinaria in the ratio of 2:2:1:1 respectively, as we wanted to obtain a wide range of phytochemicals that will eventually hit multiple disease targets. We hope that the synergistic effects of the various phytochemical from the four species will be able to be more effective in challenging the disease through its multiple targets.

Briefly, whole plant samples (minus roots) were freshly harvested, washed and dried at room temperature. The samples were cut into smaller pieces and ground with liquid nitrogen into powder and finally freeze dried. For aqueous extract, freeze-dried powder was extracted with $20 \mathrm{~mL}$ of water, $20 \mathrm{mM}$ of diethyldithiocarbamic acid and $0.5 \%$ formic acid, whereas, absolute methanol was used for methanolic extract preparation. The samples were subjected to homogenization and filtered with 15 Whatman No. 40. The extracts were stored at $-20^{\circ} \mathrm{C}$ until use and a single batch of extracts was used for all the experiments. 


\section{Identification of proteins through high performance liquid chromatography (HPLC) and mass spectrometry (LC-MS-MS) analysis}

Proteins were identified using HPLC and LC-MS-MS as previously described by Tang and colleagues [22]. For the aqueous cocktail extract, $2 \mathrm{ml}$ of supernatant was concentrated by vacuum evaporator, followed by redissolving in $20 \mathrm{mg} / \mathrm{ml}$ with $30 \%$ methanol and then subjected to LC-MS-MS. While for the methanolic extract, supernatant was subjected to rotary evaporation (Rotavapor RII, BUCHI, Switzerland) and re-dissolved with $20 \%$ methanol. The sample was then separated using solid phase extraction (SPE) column (LiChrolut RP-18 $1000 \mathrm{mg} / 6 \mathrm{ml}$, Merck Germany) with the mobile phase of $60 \%$ and $70 \%$ methanol. The collected elutes were then concentrated to a volume of $0.5 \mathrm{ml}$, then diluted eight times with $40 \%$ methanol, and finally subjected to LC-MS-MS analysis.

The separation of both extracts was carried out using HPLC binary pump, an autosampler injector compartment and a diode array detector (DAD) (1200 Series, Agilent Technologies, Germany). The reverse phase used was C-18, $150 \mathrm{~mm} \mathrm{X} 4.6 \mathrm{~mm}$ i.d. $5 \mu \mathrm{m}$ particle size of Thermo Hypersil GOLD column (Thermo Scientific, UK), while the composition of mobile phase used was $0.1 \%$ formic acid in water (solvent A) and $0.1 \%$ formic acid in acetonitrile (solvent B) with gradient for solvent B: $5 \%(5 \mathrm{~min}), 5-90 \%(60 \mathrm{~min}), 5 \%(4 \mathrm{~min})$ at a flow rate of $1 \mathrm{ml} / \mathrm{min}$. Total volume of injection was $20 \mu \mathrm{l}$ and the detection was assigned at $280 \mathrm{~nm}$ and $360 \mathrm{~nm}$. For mass spectrometry analysis, 3200 QTrap LC/MS/MS system (Applied Bioscience - MDS Sciex) was utilized. For negative ionization, the iron source was set at $500^{\circ} \mathrm{C}$ and voltage maintained at $-4.5 \mathrm{kV}$. The nitrogen generator was operated at 60 curtain gas flow, 90 psi source gas flow and 60 psi exhaust gas flow. Two scanning modes were used; enhance mass spectrometer (EMS) and enhance ion product (EPI) for a full scan mass spectra ranging $m / z 100-1200$.

\section{Virus and cell culture}

The New Guinea C (GeneBank Accession No. M29095) strain of DENV2 was obtained from the Department of Medical Microbiology, University of Malaya and used throughout the study. The virus was cultured and propagated in Aedes albopictus mosquito cell line C6/36 (ATCC: CRL-1660) with Leibowitz-15 (L-15) medium containing $1 \%$ heat-inactivated foetal bovine serum (FBS. Gibco) at $30^{\circ} \mathrm{C}$. The supernatant from infected $\mathrm{C} 6 / 36$ cells was collected for viral titre determination as well as for subsequent experimental applications. The porcine kidney (PS) cell line was maintained in L-15 media containing $5 \% \mathrm{FBS}$ at $30^{\circ} \mathrm{C}$ and the African green monkey kidney cells (Vero) (ATCC: CCR-81) were cultured in DMEM supplemented with $10 \%$ FBS at $37^{\circ} \mathrm{C}$. Cells were maintained in humidified air with $5 \% \mathrm{CO}_{2}$. Cells undergoing exponential growth were used throughout the experiments.

\section{Virus titration}

The PS cells were seeded at a cell density of $1.5 \times 10^{5}$ per well in 24-well plates (Nunc, Denmark). Ten-fold serial dilutions of viral supernatant were prepared in L15 medium containing 1\% FBS, and loaded on the cells. After 3 hours, 3\% carboxylmethyl cellulose (Calbiochem, USA) containing $3 \%$ FBS in L-15 was added into each well and further incubated for 3-4 days. After 3-4 days of incubation, the cells were washed with PBS, and stained with $1 \%$ of naphthalene blue, followed by visual counting of plaques.

\section{Determination of maximum non-toxic dose (MNTD)}

Prior to screening of Phyllanthus cocktail extracts for their antiviral properties, they were first subjected to cytotoxicity assay to identify the maximal non-toxic dose (MNTD) on Vero cells. The MNTD is a concentration where it does not show any toxicity effects on cell viability. Briefly, $1 \times 10^{4}$ Vero cells were seeded into each well of a 96-well flat-bottom plate and incubated overnight for attachment. After 24 hours, different concentrations of extracts ranging from $15.63-500 \mu \mathrm{g} / \mathrm{ml}$ were added into respective wells in triplicates and further incubated for different time points (24, 48 and 72 hours). Control wells contained cells with culture medium without the extracts and negative control wells contained only culture medium with different concentrations of the extracts. After each time course of study, $100 \mu \mathrm{l}$ of supernatant was discarded and $20 \mu \mathrm{l}$ of MTS/PMS solution (Promega, USA) was added, followed by incubation in the dark for 1 hour. Absorbance was measured using GloMax (Promega, USA) at $490 \mathrm{~nm}$ with a reference wavelength of $600 \mathrm{~nm}$. Absorbance is directly proportional to the number of live cells in the culture. Thus, the dose-response graph was presented in percentage of Vero cell's viability against different concentration of extracts.

\section{In vitro antiviral experiment}

Three different modes of treatment were performed to study the in vitro antiviral activity of Phyllanthus. For pre-treatment mode, Vero cells were seeded in a 24-well plate and incubated overnight for attachment. Before virus inoculation, the MNTD of Phyllanthus extract was added to the cells and incubated for $24 \mathrm{~h}$. DENV2 was inoculated at an MOI of 0.1 onto the Phyllanthustreated Vero cells. For the simultaneous treatment, Phyllanthus extract and DENV2 (MOI: 0.1) were prepared and then inoculated onto near confluent Vero cell 
monolayers. In the post treatment mode, DENV2 (MOI: 0.1 ) were inoculated onto the cells and incubated for $24 \mathrm{~h}$. After 24 hour, the medium was removed and replaced by the Phyllanthus extract. Subsequently for all treatment modes, the cultures were further incubated for 24,48 and $72 \mathrm{~h}$ at $37^{\circ} \mathrm{C}$ under $5 \% \mathrm{CO}_{2}$ atmosphere. After each time point of study, supernatants as well as cells were collected separately subsequent to 2 cycles of freeze-thawing and were then stored at $-80^{\circ} \mathrm{C}$ for ensuing experiments.

\section{RNA isolation and real-time RT-PCR}

Real time RT-PCR was performed to detect and quantitate viral RNA in infected Vero cells treated with Phyllanthus extract. RNA was extracted from samples (supernatant and cells) according to the instructions provided by AccuPrep viral RNA extraction Kit (Bioneer, Korea) and the extracted RNA was then stored at $-80^{\circ} \mathrm{C}$ until further use. Dengue virus standard curve was prepared using MAXIscript Transcription Kit (Ambion, USA). Ten-fold serially diluted RNA was prepared and subjected to real-time RT-PCR. The copy number of RNA was calculated based on the concentration measured and its molecular weight. RT-PCR was carried out using iScript ${ }^{\mathrm{TM}}$ One Step RT-PCR Kit with SYBR Green (Bio-Rad, USA), employing dengue group specific primers with adaption of SYBR green technology [24]. The $25 \mu$ reaction mixture containing $5 \mu$ l of sample RNA, $0.25 \mu \mathrm{l}$ of each primers, $0.25 \mu \mathrm{l}$ of reverse transcriptase enzyme, $12.5 \mu \mathrm{l}$ of SYBR Green mix and water, was amplified in a CFX96 RT-PCR machine (Bio-Rad, USA). The thermal profile for the one-step SYBR Greenbased RT-PCRs consisted of a 1 hour RT step at $45^{\circ} \mathrm{C}$, followed by 35 cycles of 3 steps amplification at $95^{\circ} \mathrm{C}$ for 30 seconds, $60^{\circ} \mathrm{C}$ for 30 seconds and 72 for 30 seconds. Fluorescence data were collected during the extension step. Negative and positive controls were included in each analytical run.

\section{Two-dimensional (2D) gel electrophoresis}

Equal amounts of total protein from (i) cells only, (ii) Phyllanthus-treated cells, (iii) DENV2- infected cells, and (iv) Phyllanthus treated-infected cells, were subjected to 2D gel electrophoresis according to the manufacturer's instructions (GE Healthcare). Briefly, total proteins were extracted from control and experimental samples by incubation with lysis buffer on ice for 30 minutes. The protein pellets were re-solubilized in rehydration solution (8 M urea, 2\% CHAPS, $40 \mathrm{mM}$ DTT, $0.5 \%$ IPG buffer pH3-11NL, bromophenol blue) and kept at $-80^{\circ} \mathrm{C}$ until further analysis. Total amount of proteins was determined using 2D Quant kit (GE Healthcare) and $250 \mu \mathrm{g}$ of proteins were rehydrated into $13 \mathrm{~cm}$ immobilized $\mathrm{pH}$ gradient (IPG) strips $(\mathrm{pH} 3-11$ nonlinear) (GE Healthcare). The first dimension was electrophoresed on the IPGphor III machine (GE Healthcare) at $20^{\circ} \mathrm{C}$ with the following settings: step 1 at $500 \mathrm{~V}$ for 1 hour; step 2 at 500-1000 V for 1 hour; step 3 at $1000-8000 \mathrm{~V}$ for 2.5 hour, and step 4 at $8000 \mathrm{~V}$ for $0.5 \mathrm{~h}$. Once first dimensional separation was completed, the gel was equilibrated as follows; first reduction with $64.8 \mathrm{mM}$ of dithiothreitol-SDS equilibration buffer (50 mM Tris- $\mathrm{HCl}$ [pH 8.8], $6 \mathrm{M}$ urea, 30\% glycerol, 2\% SDS, $0.002 \%$ bromophenol blue) for 15 minutes, followed by alkylation with $135.2 \mathrm{mM}$ of iodoacetamideSDS equilibration buffer for another 15 minutes. The second dimension electrophoresis was carried out using the SE600 Ruby system (GE Healthcare) at $25^{\circ} \mathrm{C}$ in an electrode buffer ( $25 \mathrm{mM}$ Tris, $192 \mathrm{mM}$ glycine, and $0.1 \%$ [wt/vol] SDS) with the following settings: step 1 at $100 \mathrm{~V} /$ gel for 45 minutes; step 2 at $300 \mathrm{~V} /$ gel until the run is completed. The gels used in the second dimension were $12.5 \%$ homogenous acrylamide gels casted in the laboratory. After electrophoresis, the gels were fixed with destaining solution for 30 minutes, followed by staining with hot Coomasie blue for 10 minutes. The gels were scanned using Ettan DIGE Imager (GE Healthcare). Gel images were analyzed using PDQuest 2-D Analysis Software (Bio-Rad, USA) and only protein spots which showed significant differences (more than 1.5 fold) were selected for mass spectrometry analysis.

\section{Protein digestion and desalting and MALDI-TOF/TOF analysis}

Protein spots excised from polyacrylamide gels were kept in sterile $1.5 \mathrm{ml}$ tubes and sent to Proteomics International Pty Ltd, Australia for further analysis. Briefly, the protein samples were first digested with mass spectrometry grade typsin and the peptides were extracted according to standard techniques. Peptides were analyzed by MALDI-TOF/TOF mass spectrometer using a 5800 Proteomics Analyzer [AB Sciex]. Identification of proteins was performed by using Mascot sequence matching software [Matrix Science] with Uniprot database.

\section{Data analysis}

Results were expressed as the mean \pm standard error mean (SEM) of data obtained from triplicate experiments. All data were analyzed using one way ANOVA, followed by Dunnett's test for pairwise comparison. $\mathrm{P}<$ 0.05 was considered statistically significant for all tests. All analysis was performed using SPSS software.

\section{Results}

Identification of polyphenol compounds in Phyllanthus The phytochemicals contained in all Phyllanthus species were first optimally separated using HPLC and then 
identified through LC-MS-MS. To obtain the best cocktail extract, different Phyllanthus species were mixed at specific proportions to obtain the highest amounts of health benefiting phytochemicals. The mixed cocktail extracts were analysed using both HPLC and LC-MS-MS where a variety of polyphenol compounds were identified (Figure 1). These compounds include gallic acid, galloylglucopyronside, corilagen, geraniin, rutin, quercetin glucoside, syringing, syringing diamer, digalloylglucopyronside, trigalloylglucopyronside, apigenin rhamnoside, and quercetin rhamnoside. Their individual percentages in both the aqueous and methanolic extracts are shown in Table 1 and among this compounds, geraniin constitutes the greatest amount in both the extracts, in particular in the methanolic extract.

\section{Cytotoxicity activity of aqueous and methanolic extracts of Phyllanthus}

From the cytotoxicity study using the Vero cell line, we noted that the MNTD for aqueous and methanolic extracts were $250 \mu \mathrm{g} / \mathrm{ml}$ and $15.63 \mu \mathrm{g} / \mathrm{ml}$, respectively
(Figures 2A and 2B). The aqueous cocktail extract of Phyllanthus showed lower toxicity to Vero cells as compared to the methanolic extract as its cell viability remained above $90 \%$ when treated up to $250 \mu \mathrm{g} / \mathrm{ml}$. Thus, only the aqueous cocktail extract of Phyllanthus was selected for further antiviral studies.

\section{In vitro antiviral activity of Phyllanthus}

Via real time RT-qPCR, the in vitro antiviral activities of aqueous Phyllanthus cocktail on DENV2 were assessed in 3 different treatment modes (Figure 3, Additional file 1). Comparing the three different modes of treatment, Phyllanthus showed the strongest antiviral activity against DENV2 during the simultaneous mode of treatment with more than $83-95 \%$ reduction of virus inhibition (Table 2). The morphological alterations of Vero cells due to virus replication were completely prevented when $250 \mu \mathrm{g} / \mathrm{ml}$ of the extract was added into the infected cultures. On the other hand, the virus reduction was not pronounced in pre-treatment as compared to simultaneous treatment, with only $5-46 \%$ virus inhibition

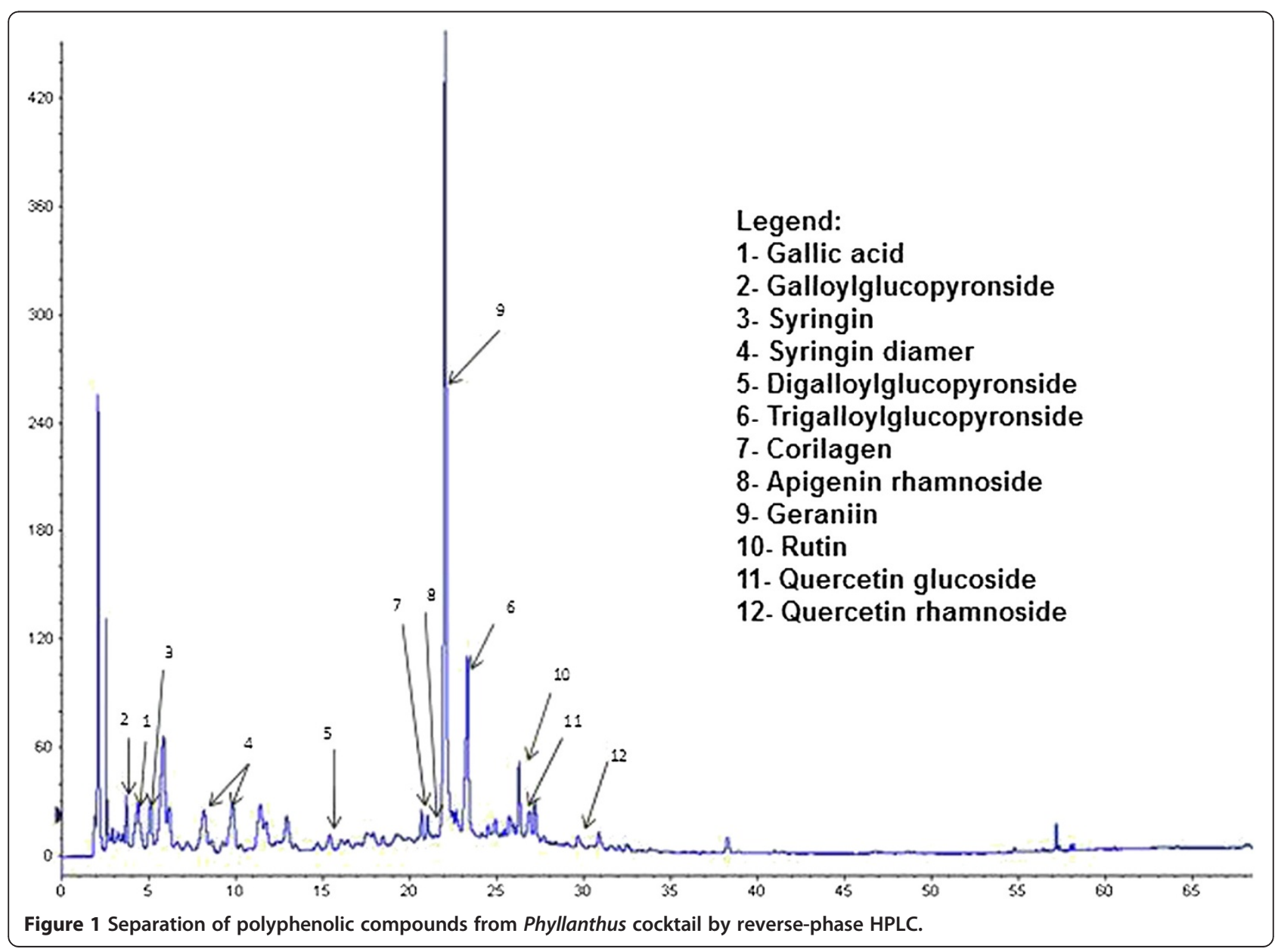


Table 1 Percentages of individual polyphenolic compound in aqueous and methanolic extracts

\begin{tabular}{llll}
\hline Location of compounds found in chromatogram & Compound & \% present in water extract & \% present in methanolic extract \\
\hline 1 & Gallic acid & 0.0004 & 0.032 \\
2 & Galloylglucopyronside & 0.0003 & Not detected \\
3 & Syringin & 0.0005 & Not detected \\
4 & Syringin diamer & 0.0005 & Not detected \\
5 & Digalloylglucopyronside & 0.0002 & 0.019 \\
6 & Trigalloylglucopyronside & 0.0003 & 0.020 \\
7 & Corilagen & 0.0008 & 0.084 \\
8 & Apigenin rhamnoside & $<0.0002$ & Not detected \\
9 & Geraniin & 3.237 & 17.140 \\
10 & Rutin & 0.012 \\
11 & Quercetin glucoside & 0.021 & 0.016 \\
12 & Quercetin rhamnoside & $<0.0002$ & Not detected \\
\hline
\end{tabular}
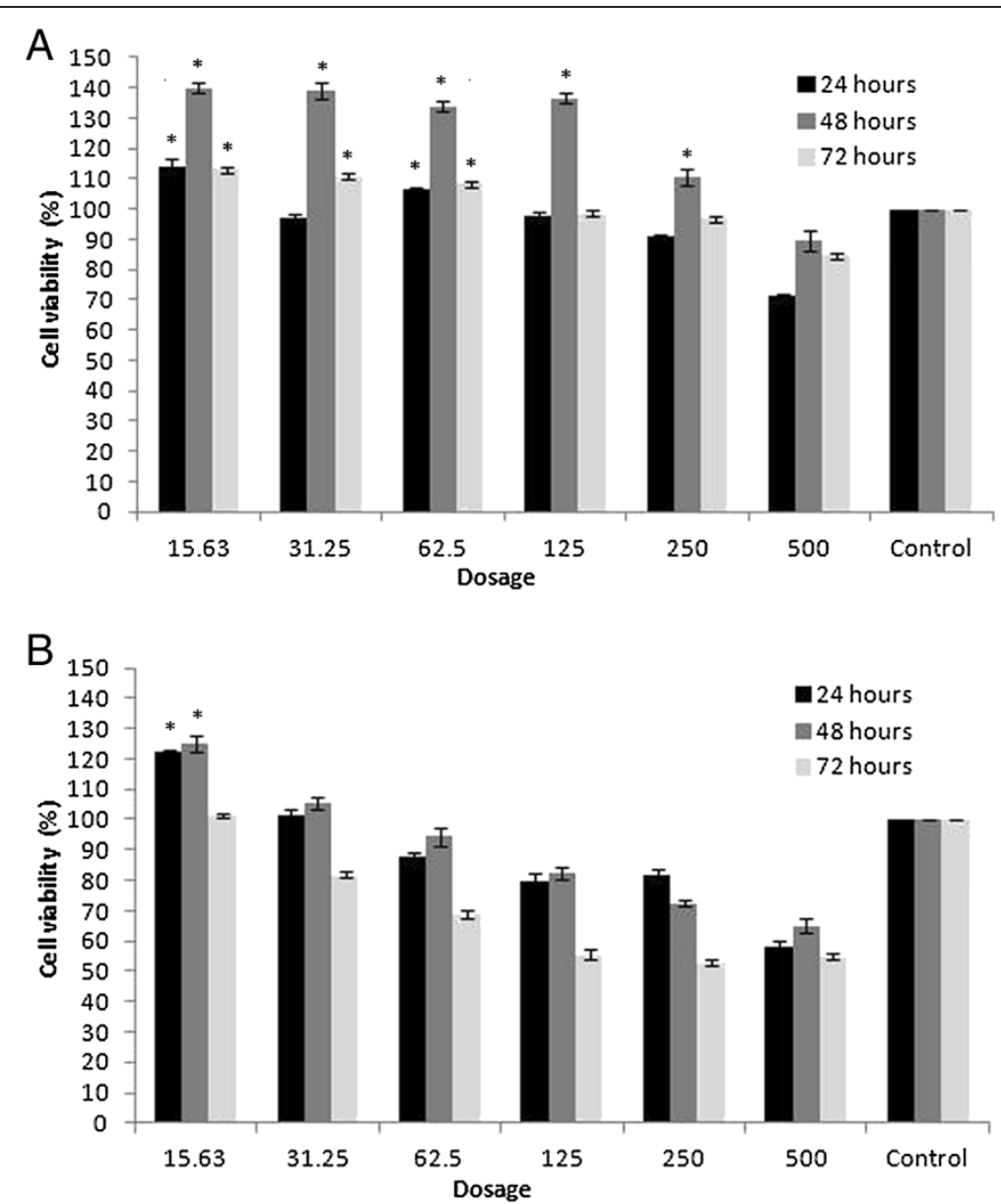

Figure 2 Percentage of Vero cells viability at different times of incubation and dosages of Phyllanthus treatments. (A) Vero cells treated with aqueous cocktail extract of Phyllanthus and (B) Vero cells treated with methanolic cocktail extract of Phyllanthus. Results are expressed as mean \pm SEM of three independent experiments. ${ }^{*}$ signifies $p<0.05$ compared to the untreated control. 

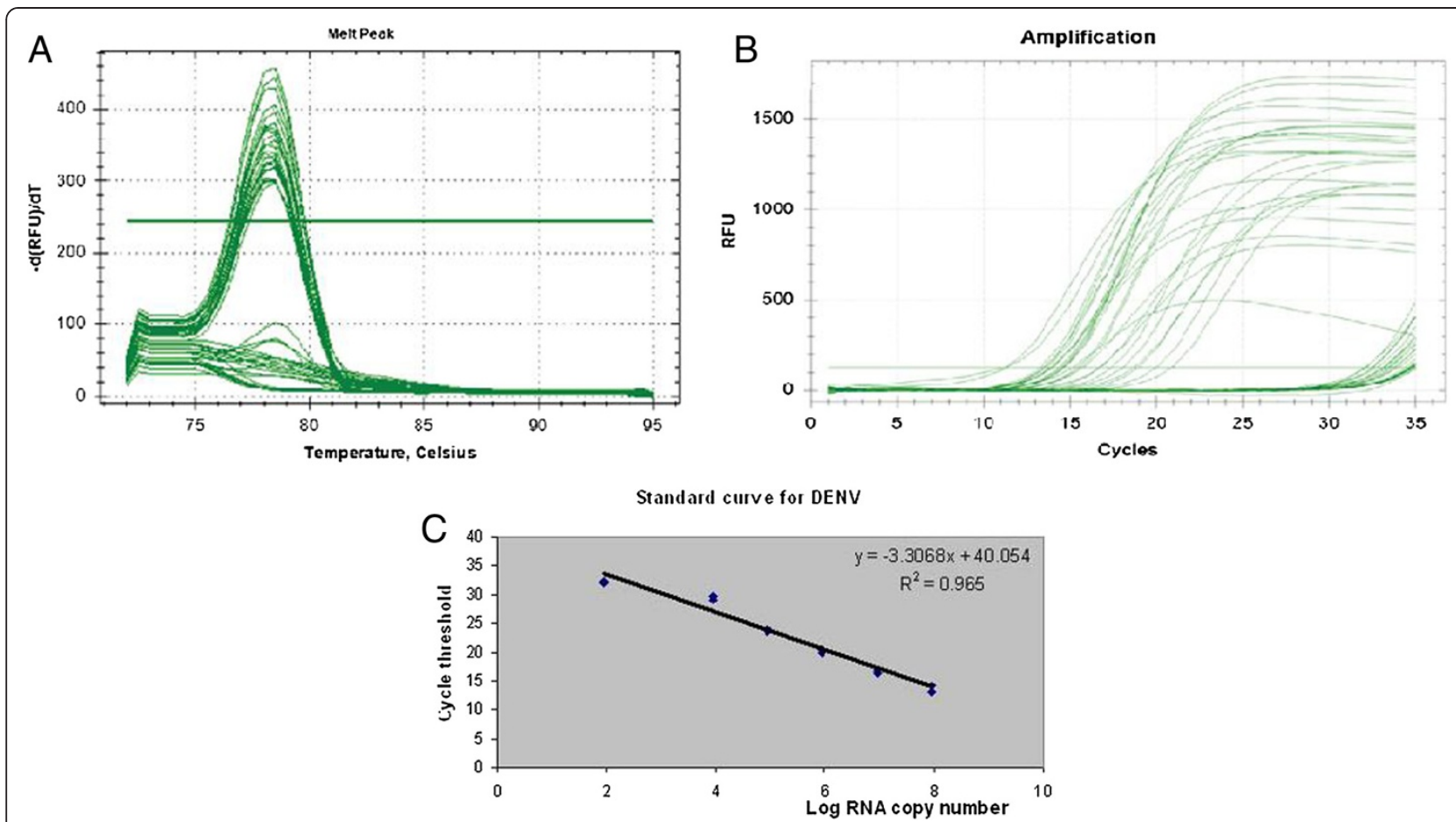

Figure 3 In-vitro antiviral assay via SYBR-Green RT-PCR. (A) Melt peak of DENV2. (B) Representative amplification plot for DENV2 antiviral assay. (C) Standard curve for DENV2.

in both supernatant and cell samples. Contrarily, no virus reduction with post-infection treatment with Phyllanthus extracts.

Proteome analysis of DENV2 infected cells with or without Phyllanthus treatment

To determine the actual antiviral mechanisms of Phyllanthus against DENV2 protein profiling of Phyllanthus-treated dengue-infected cells via 2D gel electrophoresis was carried out. This was compared to three other different protein profiles including cells only, Phyllanthus-treated cells and dengue-infected cells. A total of 52 proteins were found to be differentially expressed (Figure 4), but only 24 proteins were deemed significant (protein scores $>55$ ) after mass spectrometry analysis. Fourteen proteins which had significantly altered expression were identified, where some of these proteins are represented by more than one protein spot

Table 2 Percent Inhibition of virus copy number in cell and supernatant at three different treatment modes

\begin{tabular}{|c|c|c|c|c|c|c|}
\hline \multicolumn{7}{|c|}{ Percent Inhibition of virus copy number (\%) } \\
\hline \multicolumn{3}{|c|}{24 Hour } & \multicolumn{2}{|c|}{48 Hour } & \multicolumn{2}{|c|}{72 Hour } \\
\hline & & Supernatant & Cell & Supernatant & Cell & Supernatant \\
\hline \multicolumn{7}{|c|}{ PRE-TREATMENT } \\
\hline$C+V$ & 0 & 0 & 0 & 0 & 0 & 0 \\
\hline$C+P+V$ & $6.38 \pm 5.59$ & $46.07 \pm 0.88^{*}$ & $4.66 \pm 6.33$ & $39.01 \pm 5.01^{*}$ & 0 & $17.87 \pm 4.89^{*}$ \\
\hline \multicolumn{7}{|c|}{ SIMULTANEOUS TREATMENT } \\
\hline$C+V$ & 0 & 0 & 0 & 0 & 0 & 0 \\
\hline$C+P+V$ & $94.69 \pm 0.40^{*}$ & $82.85 \pm 3.91^{*}$ & $92.78 \pm 0.83^{*}$ & $84.61 \pm 6.58^{*}$ & $93.61 \pm 1.38^{*}$ & $91.48 \pm 3.66^{*}$ \\
\hline \multicolumn{7}{|c|}{ POST-TREATMENT } \\
\hline$C+V$ & 0 & 0 & 0 & 0 & 0 & 0 \\
\hline$C+P+V$ & 0 & 0 & 0 & 0 & 0 & 0 \\
\hline
\end{tabular}

Results are expressed as mean \pm SEM of three independent experiments. $C+V=$ dengue-infected cells; and $C+P+V=$ Phyllanthus treated-dengue infected cells. * signifies $p<0.05$. 


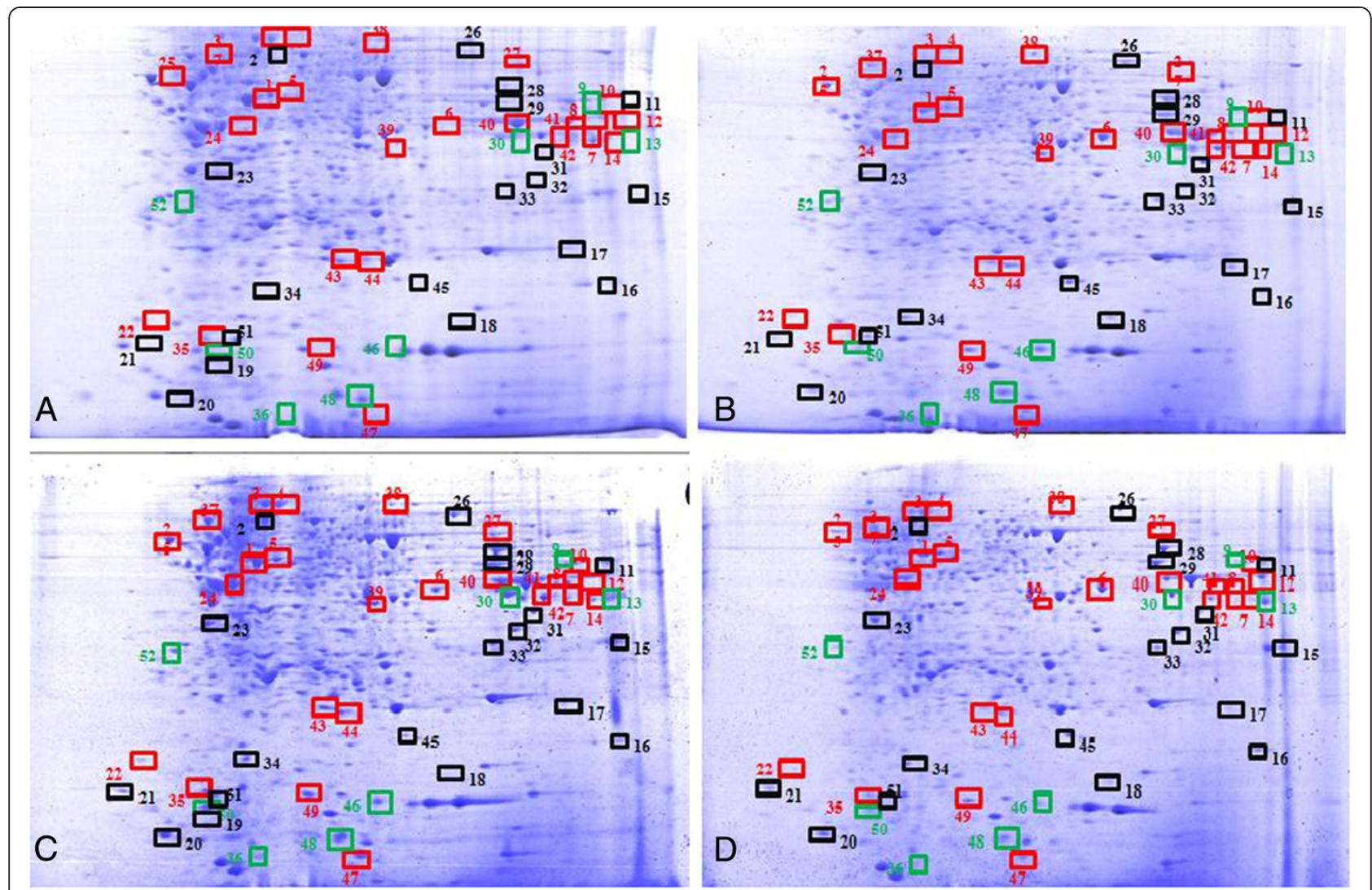

Figure 4 Proteome analysis of DENV2 infected cells with or without Phyllanthus treatment. (A) Cells Only, (B) Phyllanthus-treated cells, (C) DENV2 Infected cells and (D) Phyllanthus treated infected cells.

(Table 3). These include Calreticulin, Trim1, Heat shock $70 \mathrm{kDA}$ protein, Beta actin, Hepatocyte growth factor receptor, DNA topoisomerase I, NS3, G3PD (Glyceraldehyde-3-phosphate dehydrogenase), RBM1 (RNA-binding motif 1), Histidine triad nucleotide-binding protein 1, DNA mismatch repair protein Msh2, Chain B, Dengue virus NS2bNS3, and Polysialyltransferase. These proteins consist of host cell proteins as well as viral proteins that are involved in various biological processes, including viral entry and replication, molecular chaperoning, cytoskeletal assembly, and cellular metabolisms (Table 3; Figure 5).

\section{Discussion}

Since the discovery of Phyllanthus and its anti-hepatitis B viral effect in the late 1980s [25], the plant Phyllanthus has been tested for its various pharmacological effects. Various species of this plant genus have exhibited activities that range from being anti-hepatotoxic [26,27], antiviral $[25,28]$, antibacterial $[29,30]$, anti-diabetic $[31,32]$ to having anti-tumour and anti-carcinogenic effects $[22,23]$. Here, we assess the effectiveness of Phyllanthus against DENV which has become a global menace especially in the lack of commercialized vaccines and anti-DENV agents.

Two different Phyllanthus cocktail of different extraction method was tested initially for their toxicity against Vero cells in vitro. The aqueous cocktail displayed lower cytotoxicity even up to the concentration of $250 \mu \mathrm{g} / \mathrm{ml}$ and was thus selected for subsequent experimental procedures. The Phyllanthus cocktail had various bioactive compounds which included gallic acid, geraniin, syringing and corilage (Figure 1) $[22,23]$ and these components are believed to behave synergistically to display the anti-DENV activity observed in this study. Nevertheless, the percentages of geraniin in the extracts were the highest and hence were predicted to exert most of the antiviral effect. Yang et al. (2007) had demonstrated the suppression of herpes simplex viruses 1 and 2 by geraniin isolated from Phyllanthus urinaria [33]. Besides that, geraniin was also demonstrated to inhibit enterovirus 71 replication in both in vitro and in vivo [34]. These studies therefore accentuate the potential of Phyllanthus cocktail to act as an agent that suppresses dengue virus infection due to its presence of high geraniin content. 
Table 3 Differential protein levels in Phyllanthus-treated dengue-infected Vero cells

\begin{tabular}{|c|c|c|c|c|c|c|c|}
\hline ID & Accession & Score & Possible proteins & $\mathrm{C}$ & $C+P$ & $\mathrm{C}+\mathrm{V}$ & $C+V+P$ \\
\hline \multicolumn{8}{|c|}{ Viral Entry } \\
\hline 3 & Q28222.1 & 1059 & Heat Shock Protein 70 (Hsp70) & 192.1 & 200.2 & 312.2 & 234.3 \\
\hline 4 & Q28222.1 & 768 & Heat Shock Protein 70 (Hsp 70) & 199.5 & 287.4 & 419.3 & 282.2 \\
\hline 5 & Q2IBA6.1 & 483 & Hepatocyte Growth Factor Receptor (HGFR) & 192.7 & 200.4 & 313.4 & 259.7 \\
\hline 12 & Q2IBA6.1 & 136 & Hepatocyte Growth Factor Receptor (HGFR) & 222.5 & 0.0 & 313.2 & 218.2 \\
\hline 14 & Q2IBA6.1 & 178 & Hepatocyte Growth Factor Receptor (HGFR) & 206.3 & 0.0 & 312.5 & 200.1 \\
\hline 22 & Q2IBA6.1 & 139 & Hepatocyte Growth Factor Receptor (HGFR) & 231.9 & 237.1 & 331.3 & 225.5 \\
\hline 39 & Q2IBA6.1 & 132 & Hepatocyte Growth Factor Receptor (HGFR) & 203.8 & 206.7 & 390.0 & 212.9 \\
\hline 41 & Q2IBA6.1 & 120 & Hepatocyte Growth Factor Receptor (HGFR) & 211.0 & 224.2 & 333.2 & 221.6 \\
\hline \multicolumn{8}{|c|}{ Viral Uncoating } \\
\hline 37 & AAT48107.1 & 132 & Trim 1 & 187.4 & 198.8 & 366.1 & 535.3 \\
\hline \multicolumn{8}{|c|}{ Viral Transcription and Translation } \\
\hline 7 & ABB92440.1 & 489 & RNA Binding Motif 1 (RBMI) & 197.6 & 0.0 & 412.3 & 222.2 \\
\hline 8 & ABB92440.1 & 383 & RNA Binding Motif 1 (RBMI) & 206.0 & 0.0 & 362.1 & 226.7 \\
\hline 10 & ABB92440.1 & 141 & RNA Binding Motif 1 (RBMI) & 213.8 & 0.0 & 372.7 & 221.2 \\
\hline 24 & ABB92440.1 & 77 & RNA Binding Motif 1 (RBMI) & 180.4 & 0.0 & 362.4 & 181.7 \\
\hline 42 & ABB92440.1 & 633 & RNA Binding Motif 1 (RBMI) & 202.9 & 222.2 & 424.2 & 313.3 \\
\hline 27 & NP_739587.2 & 173 & Nonstructural protein NS3 & 0.0 & 0.0 & 312.3 & 123.2 \\
\hline 35 & 2FOM_B & 151 & Chain B, Dengue virus NS2bNS3 Protease & 0.0 & 0.0 & 262.8 & 123.2 \\
\hline 38 & Q7YR26.1 & 63 & DNA topoisomerase 1 (DNA Topo 1) & 202.9 & 211.3 & 373.2 & 229.2 \\
\hline 43 & Q5XXB5.1 & 84 & DNA mismatch repair protein Msh2 & 201.1 & 212.2 & 415.2 & 311.3 \\
\hline 44 & Q5XXB5.1 & 129 & DNA mismatch repair protein Msh2 & 209.1 & 205.3 & 394.2 & 213.3 \\
\hline 47 & Q5RF69 & 185 & Histidine triad nucleotide-binding protein 1 & 194.4 & 189.1 & 393.4 & 194.0 \\
\hline \multicolumn{8}{|c|}{ Viral Post-translational Modification } \\
\hline 25 & Q4VIT5.1 & 346 & Calreticulin & 188.0 & 200.4 & 333.3 & 213.2 \\
\hline \multicolumn{8}{|c|}{ Glucose Uptake and Glycolytic Enzymes } \\
\hline 6 & Q8CGV6 & 559 & Glyceraldehyde-3-phosphate dehydrogenase (G3PD) & 213.9 & 231.3 & 524.1 & 313.2 \\
\hline 49 & AAF17105.1 & 151 & Polysialyltransferase & 207.1 & 212.3 & 313.7 & 232.3 \\
\hline \multicolumn{8}{|c|}{ Cytoskeletal assembly } \\
\hline 1 & Q76N69.1 & 846 & Beta actin & 168.8 & 185.3 & 512.2 & 273.2 \\
\hline
\end{tabular}

$\mathrm{C}=$ cells only; $\mathrm{C}+\mathrm{P}=$ Phyllanthus-treated cells; $\mathrm{C}+\mathrm{V}=$ dengue-infected cells; $\mathrm{C}+\mathrm{P}+\mathrm{V}=$ Phyllanthus treated dengue infected cells.

The DENV replication cycle involves several steps: (1) viral attachment; (2) viral entry; (3) membrane fusion; (4) RNA release; (5) viral protein production; (6) RNA replication; (7) viral assembly; (8) viral transport and maturation and lastly (9) viral release. Hence, the antiDENV assessment of the aqueous Phyllanthus was done in a time-of-addition manner and we noted that the extract exhibited the most activity in the simultaneous assay, which was then followed by the pre-treatment assay. This implied that the Phyllanthus extract may be blocking viral adsorption into the Vero cells [35]. On the other hand, post-treatment of Vero cells following DENV infection showed minimal antiviral effects, indicating that it possibly had lower effect on the late replication stage of DENV RNA replication. Therefore, we postulated that firstly Phyllanthus could possibly be altering/blocking the virus or host factors [36] by hindering viral entry into the host cells. Secondly, this inhibition could also be partly due to the ability of some bioactive components in the extract which can directly inactivate DENV particles [37].

In order to obtain a clearer picture behind the antiDENV of Phyllanthus aqueous cocktail, we performed a protein profiling assay to study protein alterations in Phyllanthus treated DENV infected Vero cells. Of the many differentially expressed proteins observed, 13 different proteins were significantly noted to play roles in DENV reduction. As postulated, several of the proteins differentially expressed were noted to play roles in viral adsorption and entry. Host cell receptors for 


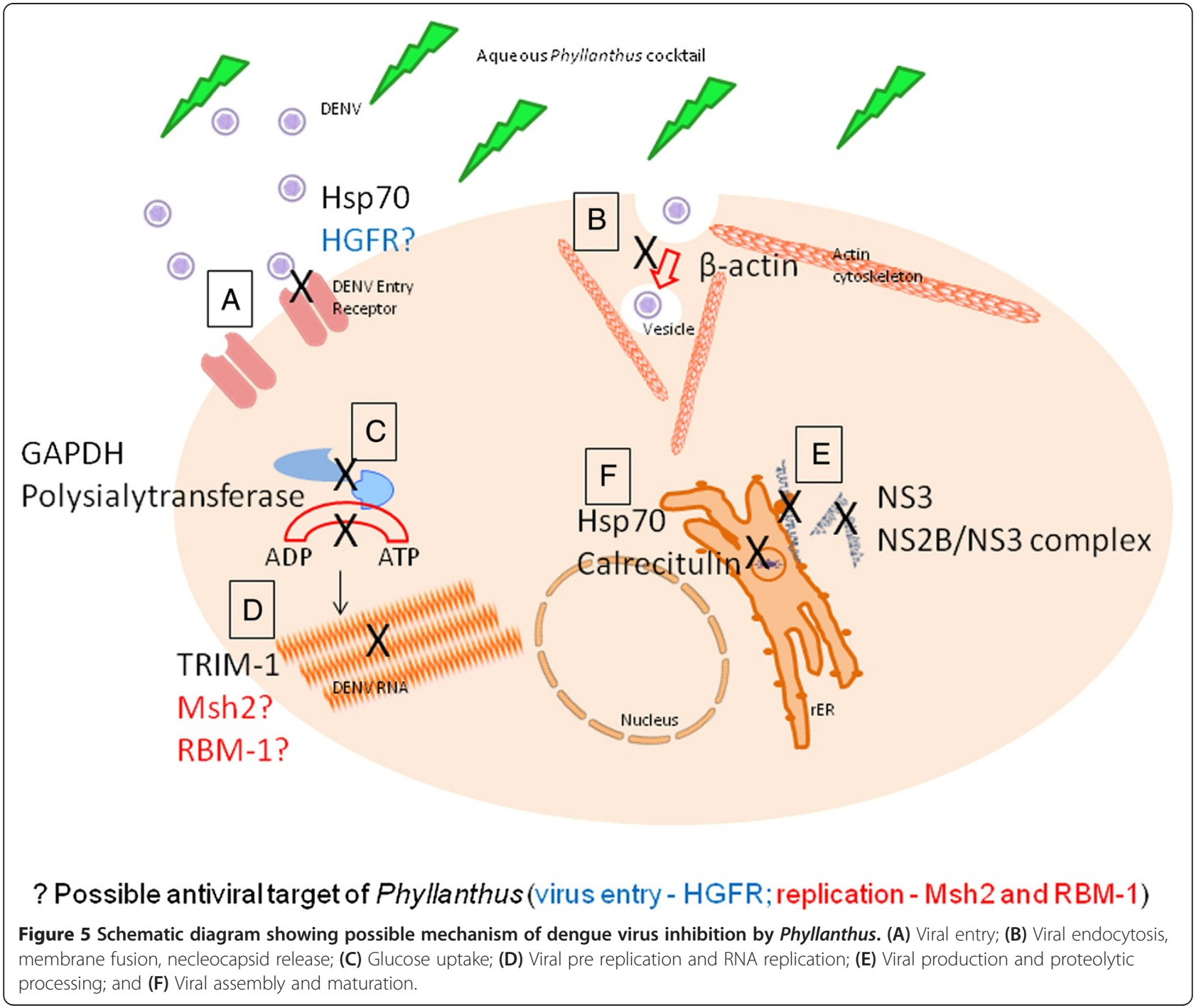

DENV include heparan sulphate; Grp78 on HepG2 cells and DC-SIGN on macrophages and monocytes. The monocytic cells also consist of Hsp70 and Hsp90 which are important components of the lipid raft in the DENV entry receptor complex [38,39]. The expression of Hsp70 was decreased in the Phyllanthus treatedDENV infected cells, indicating that viral attachment and entry via this particular receptor complex has been thwarted (Figure 5A). The Hsp70 has also been known to interact with LOX1 receptor to initiate cytotoxic $\mathrm{T}$ lymphocytes (CTL) response [40]. A reduction of Hsp70 by Phyllanthus could prevent activation of cross-reactive memory $\mathrm{T}$ cells in a secondary infection, a hypothesized mechanism behind dengue immunopathogenesis.

The hepatocytes are target cells in DENV2 infections [41] and the hepatocyte growth factor (HGF) is a mitogen for hepatocytes [42]. The HGF receptor (HGFR) has been implied as a co-receptor for adeno-associated virus type 2 infections [43], and although this has not been shown for dengue virus entry, the HGF per se has been found to be increased in the serum of dengue infected patients [44]. A down-regulation of the HGFR was noted in the Phyllanthus treated DENV2 infected cells indicating a possible role for this receptor for virus entry into cells (Figure 5A).

The cytoskeleton is an important part of a cell and one of its main components is the $\beta$-actin which was found to be down-regulated by Phyllanthus in DENV2 infected cells (Figure 5B). Dengue viruses enter cells via receptor-mediated endocytosis, form vesicles, undergo membrane fusion and their necleocapsid released into the cytoplasm. These movements are mediated by the actin skeleton [45] and in a DENV2 infection study, the actin skeleton has been shown to be an integral part for viral entry, production as well as release. Therefore, here we show that since Phyllanthus has shown inhibitory effects before and during DENV2 infection, it is clear that 
the plant may be inhibiting viral entry, viral motility in cells or the synthesis of viral polyproteins.

During a viral infection, glucose uptake and glycolytic enzyme activity is usually increased for ATP production [46], a major energy source for cells. The ATPs are necessary in a variety of ATP-dependent cellular processes during viral replication [47] and they are usually catalyzed by viral-encoded enzymes or complexes consisting of viral and host-cell proteins. Among the many enzymes involved, two (glyceraldehyde-3-phosphate dehydrogenase (GAPDH) and polysialyltransferase) were found to be down-regulated in Phyllanthus treated DENV2 infected cells (Figure 5C). The GAPDH has also been shown to co-localize with viral RNA-dependent RNA polymerase (NS5) in JEV-infected cells [48] and this may be reflected in DENV infection. The reduction of GAPDH may therefore lead to the inhibition of replication and amplification of viral RNA. Meanwhile, polysialyltransferase is an enzyme that polymerizes the sugar into a polysaccharide form and a reduction of these overexpressed enzymes in Phyllanthus-treated infected cells may lead to a low production of ATP and hence, a reduction in viral RNA replication. Generally, the decrease of both these proteins imply that Phyllanthus maybe preventing the virus from hijacking the host cell's glucose uptake and glycolytic enzymes (GAPDH and polysialyltransferase enzyme) during viral replication.

Tripartite motif (TRIM) proteins are involved in diverse cellular processes, such as cell proliferation, differentiation, oncogenesis, and apoptosis [49]. The exact functions of TRIMS during virus infection are still poorly understood but TRIM1 has been shown to display antiviral activity against $\mathrm{N}$-tropic murine leukaemia virus [50] by inhibiting the pre-reverse transcription step, suggesting a role for this particular protein against viral genome replication. Hence, it is possible that the high levels of TRIM1 protein detected in our Phyllanthus treated cells may have halted the genome replication of DENV2 and thus disrupted further viral propagation (Figure 5D).

Proteolytic processing of DENV polyprotein is an important step in viral replication and maturation. The DENV NS3 is multifunctional with a serine protease at its N-terminal, together with NS2B (NS2B/NS3 complex) cleaves the viral polyprotein for the subsequent viral assembly and maturation [51]. The dengue NS3 was detected at lower levels in Phyllanthus treated infected cells, and this directly reduced the amount of NS2B/NS3 pro complex formed as was observed in this study (Figure 5E). This complex has been a major and promising target for the development of anti-DENV agents, and Phyllanthus has shown this potential by inhibiting polyprotein processing.

Following polyprotein processing, viral budding occurs in the endoplasmic reticulum (ER), where they form immature virion and are then transported to the Golgi complex. The human immunoglobulin binding heavy chain protein, a HSP70 member and 2 ER chaperones, calnexin and calreticulin have been found to be involved in DENV E protein folding and assembly [52]. A reduction of both Hsp70 and calreticulin in our study after treatment with Phyllanthus, indicates that this plant is able to disrupt production of mature virions (Figure 5F).

Four other proteins were also detected to be downregulated by Phyllanthus in the DENV2 treated Vero cells, including DNA topoisomerase I, RNA-binding motif (RBM)-1, histidine triad nucleotide-binding protein 1, and DNA mismatch repair protein (Msh2). These proteins' functions have mainly revolved around the DNA replication of host cells, and are still elusive in the infection of DENV. Nevertheless, the Msh2 DNA mismatch protein have been shown to be required for the replication of HSV-1 and Epstein-Barr virus, where the protein has been localized to the viral replication compartments [53,54]. The RBM-1 is also known as heterogeneous ribonuclearprotein (hnRNP)- G. Several protein of the hnRNP family has been shown to up-regulated in DENV2 infected endothelial cells [55]. Phyllanthus may have been suppressing these proteins to inhibit viral replication; however this remains to be addressed.

\section{Conclusions}

From our in-vitro experiments, we noted that Phyllanthus does have antiviral activities against DENV2 and this was further supported by differential regulation of various host and viral proteins (Figure 5). We also propose that Phyllanthus is an early inhibitor as it showed most antiDENV effect prior to infection or during infection. Our suggestion is strengthened by the fact that the plant targeted 13 differentially regulated proteins which were of the cell-virus attachment, viral entry, viral polyprotein production, viral RNA replication as well as viral assembly and maturation. We envisage that the aqueous extract of Phyllanthus has the potential to be a candidate in the development of anti-dengue agents, however, additional work involving the other DENV serotypes, in vivo (toxicity and efficacy) and pre-clinical studies need to conducted for this extract to be concluded as a good anti-viral agent.

\section{Additional file}

Additional file 1: Description of Data: The in vitro viral activity of aqueous Phyllanthus cocktail at 3 different treatment modes (pre-, simultaneous-, post-) were analyzed using real time qPCR technique, whereby simultaneous mode of treatment showed strongest antiviral activity against DEN-2 virus.

Competing interests

The authors declare that they have no competing interests. 


\section{Authors' contributions}

SDS conceived of the study, participated in its design and coordination as well as helped to draft and edited the manuscript. SHL, YQT, AR, and SMW carried out the experimental works, performed the statistical analysis and drafted the manuscript. KCO, RM, and BJP performed data analysis and edited the manuscript. IBJ carried out extracts preparation and edited the manuscript. All authors read and approved the final manuscript.

\section{Acknowledgements}

This project was funded by Ministry of Science, Technology and Innovation (53-02-03-1051). University of Malaya PPP vote PV053/2011B and MARDI Grant No: 53-02-03-1002.

\section{Author details}

'Department of Medical Microbiology, Faculty of Medicine, University Malaya, Kuala Lumpur, Malaysia. ${ }^{2}$ Institute for Medical Molecular Biotechnology, University of Islamic Technology Malaysia, Kuala Lumpur, Malaysia. ${ }^{3}$ Department of Molecular Medicine, Faculty of Medicine, University of Malaya, Kuala Lumpur, Malaysia. ${ }^{4}$ Department of Trauma \& Emergency Medicine, UMMC, Kuala Lumpur, Malaysia. ${ }^{5}$ American College of Veterinary Pathology, Madison, USA. ${ }^{6}$ Biotechnology Centre, Malaysia Agricultural Research and Development Institute (MARDI), Serdang, Malaysia.

Received: 7 March 2013 Accepted: 24 July 2013

Published: 26 July 2013

\section{References}

1. Beatty ME, Stone A, Fitzsimons DW, Hanna JN, Lam SK, Vong S, Guzman MG, Mendez-Galvan JF, Halstead SB, Letson GW, et al: Best practices in dengue surveillance: a report from the Asia-Pacific and Americas Dengue Prevention Boards. PLoS Negl Trop Dis 2010, 4(11):e890.

2. Murrell S, Wu SC, Butler M: Review of dengue virus and the development of a vaccine. Biotechnol Adv 2011, 29(2):239-247.

3. Noble CG, Chen YL, Dong H, Gu F, Lim SP, Schul W, Wang QY, Shi PY: Strategies for development of Dengue virus inhibitors. Antiviral Res 2010, 85(3):450-462.

4. Steuer $\mathrm{C}$, Gege C, Fischl W, Heinonen KH, Bartenschlager R, Klein CD: Synthesis and biological evaluation of alpha-ketoamides as inhibitors of the Dengue virus protease with antiviral activity in cell-culture. Bioorg Med Chem 2011, 19(13):4067-4074.

5. Gutsche I, Coulibaly F, Voss JE, Salmon J, d'Alayer J, Ermonval M, Larquet E, Charneau P, Krey T, Megret F, et al: Secreted dengue virus nonstructural protein NS1 is an atypical barrel-shaped high-density lipoprotein. Proc Natl Acad Sci U S A 2011, 108(19):8003-8008.

6. TDR/WHO: Dengue: guidelines for diagnosis, treatment, prevention and control. Geneva: World Health Organization; 2009.

7. Halstead SB, Porterfield JS, O'Rourke EJ: Enhancement of dengue virus infection in monocytes by flavivirus antisera. AmJTrop Med Hyg 1980, 29 (4):638-642.

8. Appanna R, Huat TL, See LL, Tan PL, Vadivelu J, Devi S: Cross-reactive T-cell responses to the nonstructural regions of dengue viruses among dengue fever and dengue hemorrhagic fever patients in Malaysia. Clin Vaccine Immunol 2007, 14(8):969-977.

9. Chang J, Schul W, Yip A, Xu X, Guo JT, Block TM: Competitive inhibitor of cellular alpha-glucosidases protects mice from lethal dengue virus infection. Antiviral Res 2011, 92(2):369-371.

10. Kato D, Era S, Watanabe I, Arihara M, Sugiura N, Kimata K, Suzuki Y, Morita K, Hidari Kl, Suzuki T: Antiviral activity of chondroitin sulphate E targeting dengue virus envelope protein. Antiviral Res 2010, 88(2):236-243.

11. Jacobs M: Dengue. Medicine 2005, 33(7):18-20.

12. Piraino F, Brandt CR: Isolation and partial characterization of an antiviral, RC-183, from the edible mushroom Rozites caperata. Antiviral Res 1999, 43(2):67-78.

13. Herrmann EC Jr, Kucera LS: Antiviral substances in plants of the mint family (labiatae). 3. Peppermint (Mentha piperita) and other mint plants. Proc Soc Exp Biol Med 1967, 124(3):874-878.

14. Aswal BS, Goel AK, Kulshrestha DK, Mehrotra BN, Patnaik GK: Screening of Indian plants for biological activity: Part XV. Indian J Exp Biol 1996, 34 (5):444-467.

15. Lee-Huang S, Zhang L, Huang PL, Chang YT: Anti-HIV activity of olive leaf extract (OLE) and modulation of host cell gene expression by HIV-1 infection and OLE treatment. Biochem Biophys Res Commun 2003, 307(4):1029-1037.

16. Yamada K, Ogawa H, Hara A, Yoshida Y, Yonezawa Y, Karibe K, Nghia VB, Yoshimura $\mathrm{H}$, Yamamoto $\mathrm{Y}$, Yamada $\mathrm{M}$, et al: Mechanism of the antiviral effect of hydroxytyrosol on influenza virus appears to involve morphological change of the virus. Antiviral Res 2009, 83(1):35-44.

17. Zhu W, Chiu LC, Ooi VE, Chan PK, Ang PO Jr: Antiviral property and mechanisms of a sulphated polysaccharide from the brown alga Sargassum patens against Herpes simplex virus type 1. Phytomedicine 2006, 13(9-10):695-701.

18. Cui X, Wang Y, Kokudo N, Fang D, Tang W: Traditional Chinese medicine and related active compounds against hepatitis $B$ virus infection. Biosci Trends 2010, 4(2):39-47.

19. Cacciotti P, Libener R, Betta P, Martini F, Porta C, Procopio A, Strizzi L, Penengo L, Tognon M, Mutti L, et al: SV40 replication in human mesothelial cells induces HGF/Met receptor activation: a model for viral-related carcinogenesis of human malignant mesothelioma. Proc Natl Acad Sci U S A 2001, 98(21):12032-12037.

20. Eldeen I, Seow E, Abdullah $\mathrm{R}$, Sulaiman S: In vitro antibacterial, antioxidant, total phenolic contents and anti-HIV-1 reverse transcriptase activities of extracts of seven Phyllanthus sp. S Afr J Bot 2010, 77(1):75-79.

21. Schmelzer GH: Plant resources of tropical Africa: medicinal plants. Prota 2008, 11:270-271.

22. Tang $Y Q$, Jaganath IB, Sekaran SD: Phyllanthus spp. induces selective growth inhibition of PC-3 and MeWo human cancer cells through modulation of cell cycle and induction of apoptosis. PLoS One 2010, 5(9):e12644.

23. Lee SH, Jaganath IB, Wang SM, Sekaran SD: Antimetastatic effects of Phyllanthus on human lung (A549) and breast (MCF-7) cancer cell lines. PLoS One 2011, 6(6):e20994.

24. Yong YK, Thayan R, Chong HT, Tan CT, Sekaran SD: Rapid detection and serotyping of dengue virus by multiplex RT-PCR and real-time SYBR green RT-PCR. Singapore Med J 2007, 48(7):662-668.

25. Venkateswaran PS, Millman I, Blumberg BS: Effects of an extract from Phyllanthus niruri on hepatitis $B$ and woodchuck hepatitis viruses: in vitro and in vivo studies. Proc Natl Acad Sci U S A 1987, 84(1):274-278.

26. Zhou S, Xu C, Zhou N, Huang Y, Huang L, Chen X, Hu Y, Liao Y: Mechanism of protective action of Phyllanthus urinaria L. against injuries of liver cells]. Zhongguo Zhong yao za zhi = Zhongguo zhongyao zazhi = China journal of Chinese materia medica 1997, 22(2):109.

27. Rajeshkumar NV, Kuttan R: Phyllanthus amarus extract administration increases the life span of rats with hepatocellular carcinoma. J Ethnopharmacol 2000, 73(1-2):215-219.

28. Ott M, Thyagarajan SP, Gupta S: Phyllanthus amarus suppresses hepatitis $B$ virus by interrupting interactions between $\mathrm{HBV}$ enhancer I and cellular transcription factors. Eur J Clin Invest 1997, 27(11):908-915.

29. Mazumder A, Mahato A, Mazumder R: Antimicrobial potentiality of Phyllanthus amarus against drug resistant pathogens. Nat Prod Res 2006, 20(4):323-326.

30. Kloucek P, Polesny Z, Svobodova B, Vlkova E, Kokoska L: Antibacterial screening of some Peruvian medicinal plants used in Calleria District. J Ethnopharmacol 2005, 99(2):309-312.

31. Mazunder UK, Gupta M, Rajeshwar Y: Antihyperglycemic effect and antioxidant potential of Phyllanthus niruri (Euphorbiaceae) in streptozotocin induced diabetic rats. Eur Bull Drug Res 2005, 13(1):15-23.

32. Raphael KR, Sabu MC, Kuttan R: Hypoglycemic effect of methanol extract of Phyllanthus amarus Schum \& Thonn on alloxan induced diabetes mellitus in rats and its relation with antioxidant potential. Indian J Exp Biol 2002, 40(8):905-909.

33. Yang $C M$, Cheng HY, Lin TC, Chiang LC, Lin CC: The in vitro activity of geraniin and 1,3,4,6-tetra-O-galloyl-beta-D-glucose isolated from Phyllanthus urinaria against herpes simplex virus type 1 and type 2 infection. J Ethnopharmacol 2007, 110(3):555-558.

34. Yang $Y$, Zhang L, Fan $X$, Qin C, Liu J: Antiviral effect of geraniin on human enterovirus 71 in vitro and in vivo. Bioorg Med Chem Lett 2012, 22(6):22092211.

35. Kwon H-J, Kim H-H, Yoon S, Ryu Y, Chang J, Cho K-O, Rho M-C, Park S-J, Lee W: In Vitro inhibitory activity of Alpinia katsumadai extracts against influenza virus infection and hemagglutination. Virol J 2010, 7(1):307.

36. Sepulveda CS, Garcia CC, Fascio ML, D'Accorso NB, Docampo Palacios ML, Pellon RF, Damonte EB: Inhibition of Junin virus RNA synthesis by an antiviral acridone derivative. Antiviral Res 2012, 93(1):16-22. 
37. Alvarez AL, del Barrio G, Kouri V, Martinez PA, Suarez B, Parra F: In vitro antiherpetic activity of an aqueous extract from the plant Phyllanthus orbicularis. Phytomedicine 2009, 16(10):960-966.

38. Jindadamrongwech S, Thepparit C, Smith D: Identification of GRP 78 (BiP) as a liver cell expressed receptor element for dengue virus serotype 2 . Arch Virol 2004, 149(5):915-927.

39. Chavez-Salinas S, Ceballos-Olvera I, Reyes-del Valle J, Medina F, del Angel RM: Heat shock effect upon dengue virus replication into U937 cells. Virus Res 2008, 138(1-2):111-118.

40. Delneste Y, Magistrelli G, Gauchat J-F, Haeuw J-F, Aubry J-P, Nakamura K, Kawakami-Honda N, Goetsch L, Sawamura T, Bonnefoy J-Y, et al: Involvement of LOX-1 in dendritic cell-mediated antigen crosspresentation. Immunity 2002, 17(3):353-362.

41. Suksanpaisan L, Cabrera-Hernandez A, Smith DR: Infection of human primary hepatocytes with dengue virus serotype 2. J Med Virol 2007, 79 (3):300-307.

42. Ishiki Y, Ohnishi H, Muto Y, Matsumoto K, Nakamura T: Direct evidence that hepatocyte growth factor is a hepatotrophic factor for liver regeneration and has a potent antihepatitis effect in vivo. Hepatology 1992, 16(5):1227-1235.

43. Kashiwakura $Y$, Tamayose $K$, Iwabuchi $K$, Hirai $Y$, Shimada T, Matsumoto $K$, Nakamura T, Watanabe M, Oshimi K, Daida H: Hepatocyte growth factor receptor is a coreceptor for adeno-associated virus type 2 infection. J Virol 2005, 79(1):609-614.

44. Voraphani N, Theamboonlers A, Khongphatthanayothin A, Srisai C, Poovorawan Y: Increased level of hepatocyte growth factor in children with dengue virus infection. Ann Trop Paediatr 2010, 30(3):213-218.

45. McNiven MA, Thompson HM: Vesicle formation at the plasma membrane and trans-Golgi network: the same but different. Science 2006, 313 (5793):1591-1594.

46. El-Bacha T, Menezes MM, Azevedo e Silva MC, Sola-Penna M, Da Poian AT: Mayaro virus infection alters glucose metabolism in cultured cells through activation of the enzyme 6-phosphofructo 1-kinase. Mol Cell Biochem 2004, 266(1-2):191-198.

47. Ranji A, Boris-Lawrie K: RNA helicases: emerging roles in viral replication and the host innate response. RNA Biol 2010, 7(6):775-787.

48. Yang SH, Liu ML, Tien CF, Chou SJ, Chang RY: Glyceraldehyde-3-phosphate dehydrogenase (GAPDH) interaction with 3 ' ends of Japanese encephalitis virus RNA and colocalization with the viral NS5 protein. J Biomed Sci 2009, 16:40.

49. Nisole S, Stoye JP, Saib A: TRIM family proteins: retroviral restriction and antiviral defence. Nat Rev Microbiol 2005, 3(10):799-808.

50. Yap MW, Nisole S, Lynch C, Stoye JP: Trim5a protein restricts both HIV-1 and murine leukemia virus. Proc Natl Acad Sci U S A 2004, 101(29):10786.

51. Nitsche C, Behnam MA, Steuer C, Klein CD: Retro peptide-hybrids as selective inhibitors of the Dengue virus NS2B-NS3 protease. Antiviral Res 2012, 94(1):72-79.

52. Limjindaporn T, Wongwiwat W, Noisakran S, Srisawat C, Netsawang J, Puttikhunt C, Kasinrerk W, Avirutnan P, Thiemmeca S, Sriburi R, et al: Interaction of dengue virus envelope protein with endoplasmic reticulum-resident chaperones facilitates dengue virus production. Biochem Biophys Res Comm 2009, 379(2):196-200.

53. Daikoku T, Kudoh A, Sugaya Y, Iwahori S, Shirata N, Isomura H, Tsurumi T: Postreplicative mismatch repair factors Are recruited to Epstein-Barr virus replication compartments. J Biol Chem 2006, 281(16):11422-11430.

54. Mohni KN, Mastrocola AS, Bai P, Weller SK, Heinen CD: DNA mismatch repair proteins Are required for efficient herpes simplex virus 1 replication. J Virol 2011, 85(23):12241-12253.

55. Kanlaya R, Pattanakitsakul S-n, Sinchaikul S, Chen S-T, Thongboonkerd V: Vimentin interacts with heterogeneous nuclear ribonucleoproteins and dengue nonstructural protein 1 and is important for viral replication and release. Mol BioSys 2010, 6(5):795-806.

doi:10.1186/1472-6882-13-192

Cite this article as: Lee et al.: Effects of cocktail of four local Malaysian medicinal plants (Phyllanthus spp.) against dengue virus 2. BMC Complementary and Alternative Medicine 2013 13:192.

\section{Submit your next manuscript to BioMed Central and take full advantage of:}

- Convenient online submission

- Thorough peer review

- No space constraints or color figure charges

- Immediate publication on acceptance

- Inclusion in PubMed, CAS, Scopus and Google Scholar

- Research which is freely available for redistribution

Submit your manuscript at www.biomedcentral.com/submit
C Biomed Central 